\title{
Commissioning of the ATLAS Liquid Argon Calorimeter
}

\author{
S. Laplace ${ }^{\mathrm{a}, *}$, \\ on behalf of the ATLAS Liquid Argon Calorimeter Group \\ ${ }^{a}$ LAPP, Université de Savoie, CNRS/IN2P3, Annecy-Le-Vieux, France
}

\begin{abstract}
The in-situ commissioning of the ATLAS liquid argon calorimeter is taking place since three years. During this period, it has been fully tested by means of frequent calibration runs, and the analysis of the large cosmic muon data samples and of the few beam splash events that occurred on September 10th, 2008. This has allowed to obtain a stable set of calibration constants for the first collisions, and to measure the in-situ calorimeter performances that were found to be at the expected level.
\end{abstract}

Key words: Calorimeter, Liquid Argon, LHC, ATLAS

\section{Introduction}

The construction and installation of the liquid argon (LAr) calorimeter and its readout system were completed in March 2006. Since then, the calibration and readout systems have been extensively used by taking very frequent calibration runs. Physics data coming from cosmic muons (since summer 2006 up to now) and from the first LHC beam events (September 10 to 12 th, 2008) were analyzed and used to measure the calorimeter in-situ performance.

\section{The Liquid Argon Calorimeter}

The ATLAS LAr calorimeters consist of four calorimeters located in three cryostats filled with liquid argon which acts as active medium [1]. The passive material and the geometry are specific to each part, and are detailed below:

- the electromagnetic barrel and endcap calorimeters (EMB and EMEC) provide a precise measurement of electron and photon positions and energies up to a pseudo rapidity of 3.2. Their absorbers are made of lead, achieving a minimal radiation length of $22 X_{0}$. Their specific accordion geometry ensures a full $\phi$ hermiticity and a uniform and fast response. They are segmented in three longitudinal compartments (called the strip, middle and back samplings) to extract the shower shape, as well as a presampler layer in order to estimate the loss due to the dead material in front of the calorimeter. The resolution is expected to be $\sigma(E) / E=10 \% / \sqrt{E} \oplus 0.7 \%$.

- the hadronic endcap (HEC) is a classical sandwich calorimeter with copper as passive material. Its pseudo rapidity coverage ranges from 1.5 to 3.2 and it is also segmented in four longitudinal compartments. The resolution for hadrons is expected to be $\sigma(E) / E=50 \% / \sqrt{E} \oplus 3 \%$.

\footnotetext{
${ }^{*}$ Corresponding author

Email addresses: laplace@lapp.in2p3.fr (S. Laplace)
}

- the forward calorimeter (FCAL) detects the particles in the forward region with a pseudo rapidity coverage between 3.2 and 4.8. Due to the high particles occupancy in this region, a specific geometry with very thin liquid argon gaps (between $250 \mathrm{~m}$ and $500 \mathrm{~m}$ ) has been adopted to limit the space charge, what could induce detection inefficiencies. The absorbers are made of tungsten (in the first compartment) or copper (in the second and third compartments). The resolution for hadrons is expected to be $\sigma(E) / E=100 \% / \sqrt{E} \oplus 10 \%$.

The three sub-calorimeters share the same readout electronics: the signal read from the electrodes is first gathered into cells (a cell gathers four electrodes along the $\phi$ direction) by means of summing boards located on mother boards. It is then processed by the Front End Board [2] (FEB) hosted just outside the cryostats. The raw triangular ionization signal is amplified and split into three linear gain scales in the ratio $1 / 10 / 100$. To optimize the signal-to-noise ratio, the signal is shaped by a bipolar $C R-(R C)^{2}$ filter. It is then sampled at the LHC bunch-crossing frequency of $40 \mathrm{MHz}$ and stored in pipelines during the L1 latency. For events accepted by the L1 trigger, five samples in one gain scale are read from the pipeline and digitized by 12bit ADCs, to be finally sent by optical fibers to the back-end electronics [3] housed $70 \mathrm{~m}$ away from the detector.

The back-end system digitally processes the data coming from the FEB. It performs various data integrity checks and higher level monitoring tasks, but most importantly, it applies an optimal filtering algorithm to the samples in order to compute the energy and timing of every calorimeter cell.

A calibration signal [4] with an exponential shape can be injected to the mother boards in order to monitor the electronics response and to compute the electronic gain. Because the injected calibration signal has a different shape than the ionization one (exponential versus triangular), and because it is injected at a different point (mother boards versus electrodes), the pulse shape that is measured at the FEB exit differs in calibration and 
physics modes. Several methods [5, 6, 7] allow to modelize this difference by predicting the ionization pulse shape starting from the calibration one: it is this predicted pulse shape that is used to compute optimal filtering coefficients.

\section{Calorimeter Performances}

\subsection{High energy deposits}

The first beam splash events have been used to check high energy depositions in the LAr calorimeter. Figure 1 shows the projected energy along the $\phi$ axis for $-0.8<\eta<0$ for the different LAr barrel calorimeter layers. Only cells with an energy above $5 \sigma$ above noise are considered. The eight-fold structure reflects the endcap toroid matter at high radius (S2 and S3), and the sixteen-fold structure shows the additional matter and shielding at low radius (PS and S1).

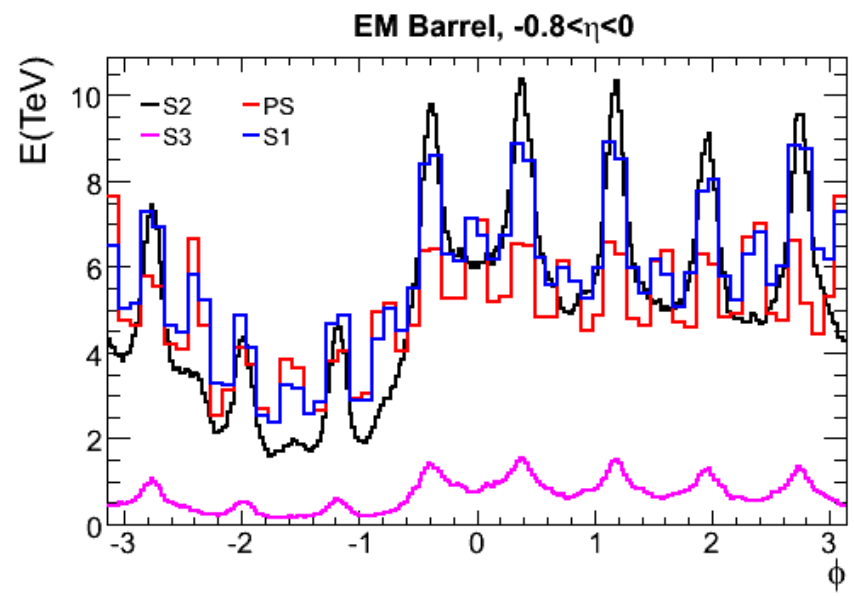

Figure 1: Energy deposition in the LAr calorimeter barrel in each sampling (PS $=$ presampler, $\mathrm{S} 1=$ front, $\mathrm{S} 2=$ middle, $\mathrm{S} 3=$ back) projected along the $\phi$ axis.

\subsection{Timing Alignment}

Beam splash events also allow to check the cell timing and derive timing delays for the first collisions. Figure 2 shows the comparison between the predicted and measured cell timings averaged over all front-end crates as a function of the FEB slot in the crate. The measured timings are obtained using the optimal filtering coefficients and are corrected by a time-of-flight correction to make as if the particles were coming from the collision point. The predicted timings are derived from the calibration timings taking into account the different cable lengths involved in the readout path. The agreement between the two timings is better than \pm 2 ns for most of the slots. The residual disagreements can be corrected using a programmable delay on each FEB.

\subsection{Ionization Pulse Shape}

The prediction of the ionization pulse shape has been successfully validated in past testbeams. Cosmic muons and first LHC beam can also be used to check this prediction in-situ. The comparison between the predicted and measured pulse shapes

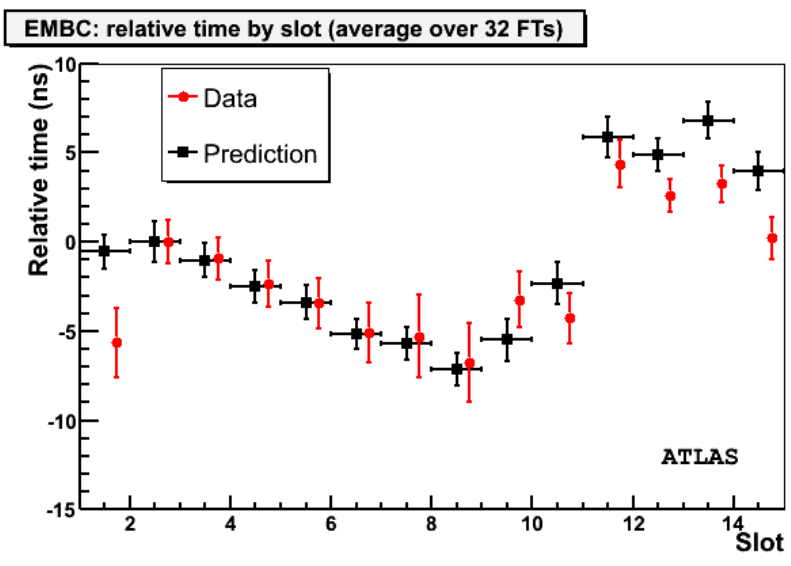

Figure 2: Comparison between the predicted (black squares) and measured (red dots) cell timings for each FEB slot in the front end crate, averaged over all front-end crates.

in the electromagnetic LAr calorimeter using cosmic muons is shown in Fig. 3. An agreement at the level of $2 \%$ is observed in this randomly chosen cell, but a more global study shows that the agreement ranges from $2 \%$ in the barrel to $4 \%$ in the endcap calorimeter.

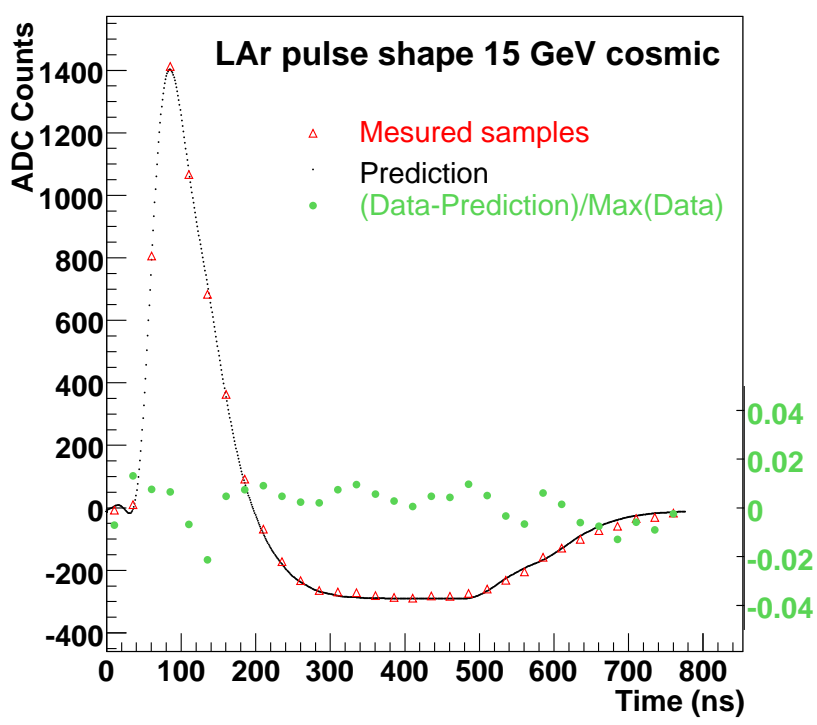

Figure 3: Comparison between the predicted (black dots) and measured (red triangles) pulse shape samples in cosmic muons data. The green dots show the normalized difference between the two.

\subsection{Calorimeter Uniformity}

The response uniformity [8] of the electromagnetic barrel calorimeter was measured in-situ using cosmics muons data collected between August 2006 and March 2007. In these data, only nine calorimeter modules were readout, and no inner detector information was available. The very small energy deposition of the muon (only due to ionization) is reconstructed using two different clusters: a so-called " $3 \times 3$ " cluster gathering the 
cells in all samplings falling inside a square of $3 \times 3$ in middle cell unit around a seed cell, and a "LArMuID" cluster that gathers a few cells based on signal-to-noise values. The energy distributions of these two clusters are then fit with a Landau function that accounts for the ionization energy deposition fluctuations, convoluted with a Gaussian function that accounts for the detector resolution. The Most Probable Value (MPV) of the Landau is plotted as a function of $\eta$ for both Monte Carlo (MC) and data in Fig. 4. The 10\% variation of the energy deposition along $\eta$ is due to the different cell depths: since the muon energy deposition is proportionnal to the path length in the calorimeter, the landau MPV naturally follows the cell depth variations. The difference between the MC and data MPV distributions quantifies the response uniformity which is found to be of the order of $2 \%$.

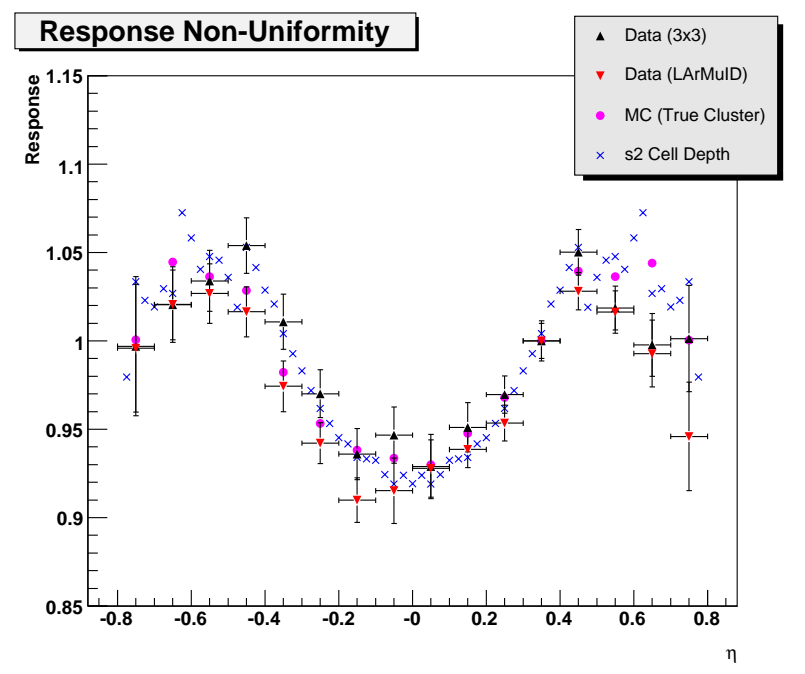

Figure 4: Most probable value from the landau fit at different $\eta$ positions: the black and red triangles show the data, reconstructed with two different clusters (see text). The red dots show the Monte Carlo response, while the blue crosses represent the average cell depth at a given $\eta$ position.

\subsection{Missing Transverse Energy}

Random triggers can be used to check the missing transverse energy $\left(E_{T}^{\text {miss }}\right)$ resolution on events that should have no $E_{T}^{\text {miss }}$. The missing transverse energy is computed using two noisesuppression methods: in the first method, all calorimeter cells that have a signal that is $2 \sigma$ above noise are kept. Since this threshold is low, one expects to create a fake $E_{T}^{\text {miss }}$ by picking up the upward noise fluctuations. In the second method, a more refined "4-2-0" topological cluster algorithm is used: the clustering starts from cells which a signal-to-noise ratio greater than 4 . All cells around the seed that have a signal-to-noise ratio above 2 are added to the cluster. Finally, all neighboring cells of the cluster are also added (they correspond to the last 0 signal-to-noise threshold of the "4-2-0" configuration). As shown on Fig. 5, which displays the $E_{T}^{\text {miss }}$ for these two methods, this second algorithm leads to much less fake $E_{T}^{\text {miss }}$.

The measured $E_{T}^{\text {miss }}$ is compared to the expected distribution obtained from a randomization of the cell energy with a

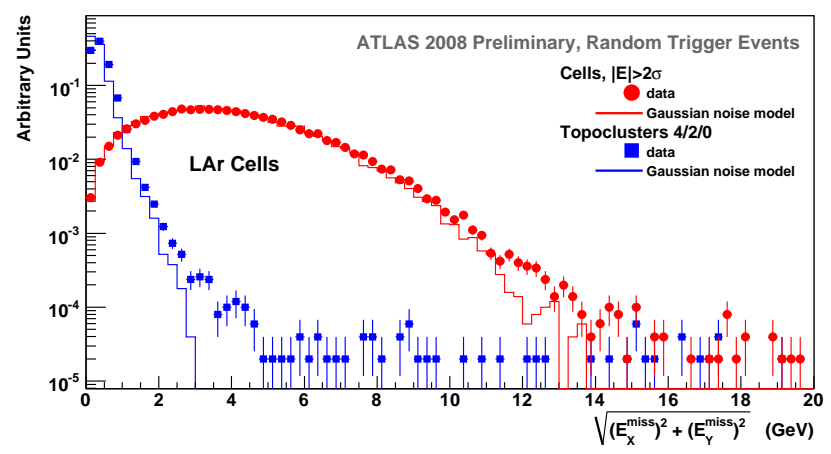

Figure 5: Distributions of missing transverse energy for the two noisesuppression methods (see text). The measured points (dots) are compared with the predicted $E_{T}^{\text {miss }}$ (full lines).

Gaussian noise (for each cell, energy values are picked by this Gaussian distribution which is centered at 0 and has an standard deviation which is equal to the respective $\sigma$ noise value). The measured $E_{T}^{\text {miss }}$ agrees well with the expectation, except for the presence of a tail at larger $E_{T}^{\text {miss }}$ value, which has been understood and corrected since then 1 .

\subsection{Electrons from Ionization}

A search for electrons coming from delta rays caused by cosmics muons has been performed. To start with, 3.5 million events that have a track reconstructed at the trigger level 2 are considered. A loose $\phi$ track matching is performed with electromagnetic clusters that have a transverse energy above 3 GeV: 11000 candidates remain at this stage. The cluster lateral shower shapes in the first and second calorimeter samplings are required to be consistent with those from an electron. Finally, the associated track is required to have at least 25 Transition Radiation Tracker (TRT) hits 2 , and the remaining events are split in two categories:

- 1229 events with only one reconstructed track which are expected to correspond to Bremsstrahlung photons coming from the muon.

- 85 events with at least two reconstructed tracks which are considered as ionization electron candidates.

Two variables are used to understand the background contamination coming from the Bremsstrahlung events to the signal electron candidates: the ratio $E / p$ of the energy $E$ reconstructed in the calorimeter and the momentum $p$ reconstructed in the tracker ( $E / p$ is around 1 for electrons), and the ratio of high-threshold over the low-threshold TRT hits, $N_{\text {TRT }}^{\text {high/low }}$, which is expected to be larger for relativistic particles such as

\footnotetext{
${ }^{1}$ An unexpected source of noise coming from the filtering box on the high voltage lines of some PS modules was fixed.

${ }^{2}$ The TRT is a drift-tube detector that is part of the ATLAS tracking system. It also provides particle identification between electrons and pions/muons by making use of transition radiation produced by relativistic particles such as electrons.
} 
electrons than for other non-relativistic particles such as muons. A signal box is defined in these two parameters space, with limits that vary as a function of $\eta$ and $p_{T}$. For example, at low $\eta$, the limits are $0.8<E / p<2.5$ and $N_{T R T}^{\text {high } / \text { low }}>0.8$. Less than $2 \%$ (19 of the 1229) of the Bremsstrahlung candidates fall into the signal box, while more than $40 \%$ (36 out of 85 ) of the electron candidates satisfy the same criteria.

A two-dimensional maximum likelihood fit is performed over these two variables to evaluate the background contribution to the signal box: the projected distributions onto the two variables are shown on Fig. 6 and 7 The background probability distribution function is taken from the Bremsstrahlung sample. Clear excesses are seen in the signal regions indicating the presence of ionization electrons. From the fit, the number of background events in the signal box is estimated to be $8.7 \pm 3.1$.

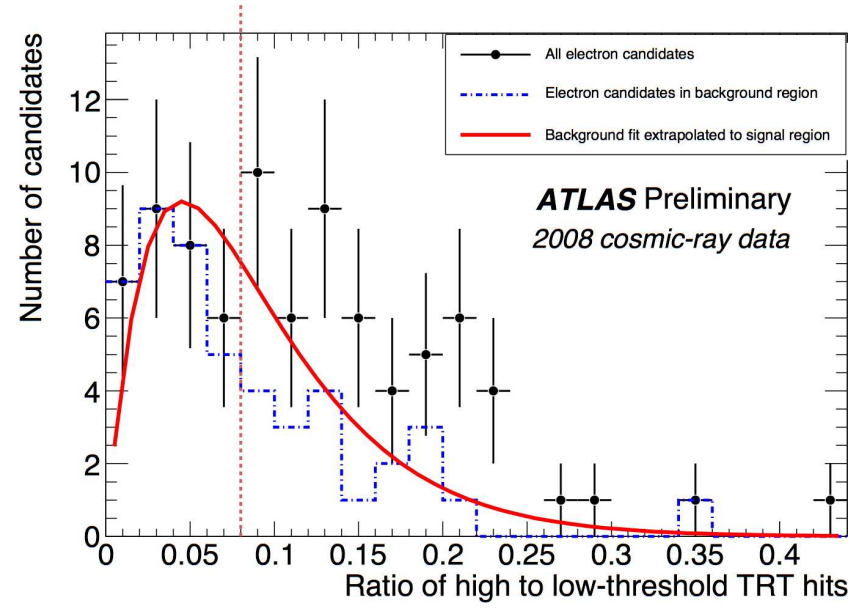

Figure 6: Ratio of high-threshold over low-threshold TRT hits for all electron candidates (dots). The red line shows the expected background distribution.

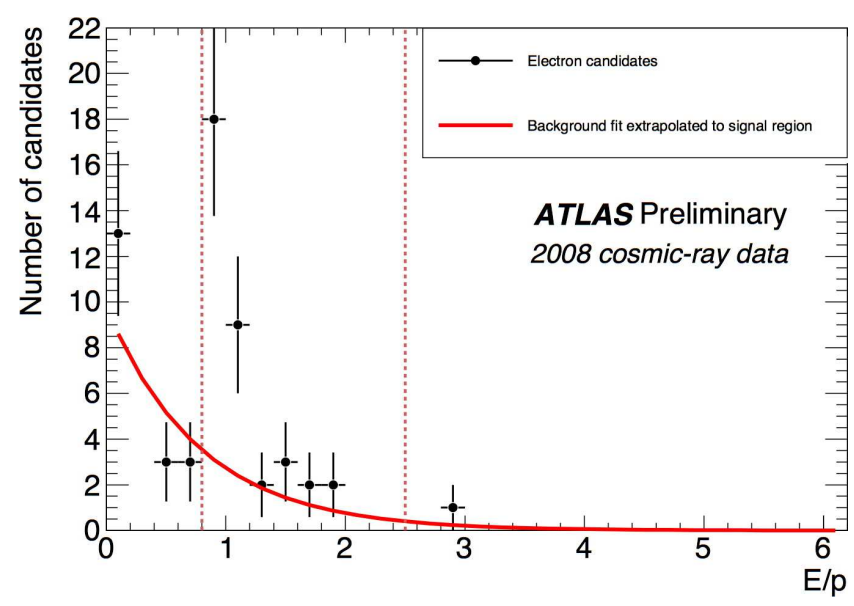

Figure 7: $E / p$ for all electron candidates (dots). The red line shows the expected background distribution.

The events remaining in the signal box 3 are further studied: a comparison of the measured shower shapes and simu-

\footnotetext{
${ }^{3}$ Over the 36 events remaining, 4 have a positive charge and are removed.
}

lated ones 4 is shown on Fig. 8 a good agreement between data and Monte Carlo is observed.
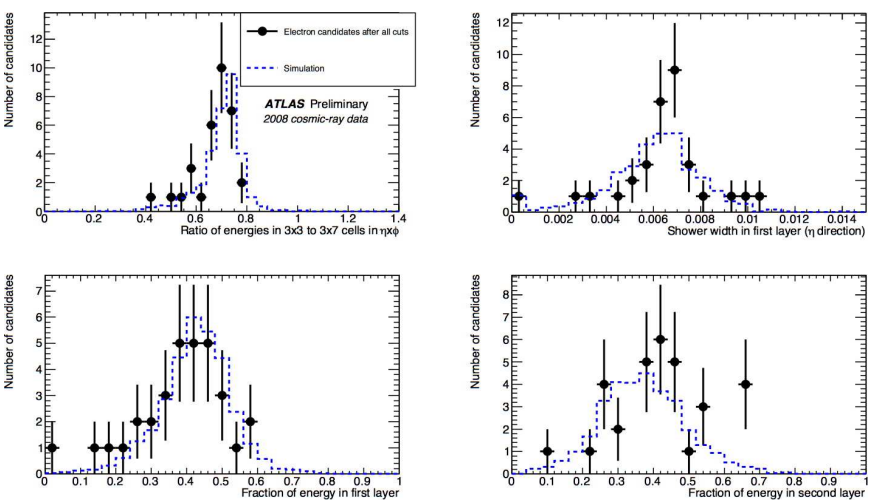

Figure 8: Comparison of shower shapes between electron candidates in the cosmics data sample and a Monte Carlo simulation of projective electrons with a transverse energy of $5 \mathrm{GeV}$.

\section{Conclusion}

The liquid argon calorimeters of ATLAS are installed in the cavern since 2006. After 10 years of development, several beam tests and in situ commissioning with cosmic muons, they were fully operational for the first runs of LHC commissioning in September 2008. Results obtained from cosmics muons and single beam events are promising, and the first electrons were observed in the electromagnetic calorimeter.

\section{Acknowledgments}

The work presented here has been performed within the ATLAS LAr collaboration. It would not have been possible without the dedicated effort of many people in our LAr detector group and the ATLAS collaboration over many years. I am especially indebted to people who built, integrated and installed the LAr detectors in the ATLAS cavern and those who operate the detector on a daily basis.

\section{References}

[1] G. Aad et al. [ATLAS Collaboration], JINST 3 (2008) S08003.

[2] N. J. Buchanan et al., JINST 3 (2008) P09003.

[3] A. Bazan et al. [Liquid Argon Back End Electronics Collaboration], JINST 2 (2007) P06002.

[4] J. Colas et al., Nucl. Instrum. Meth. A 593 (2008) 269.

[5] Collard, C et al, ATL-LARG-PUB-2007-0010

[6] Prieur, D , ATL-LARG-PUB-2005-001

[7] Banfi, D et al, J. Instrum. 1 (2006) P08001

[8] Cooke, M et al, ATL-LARG-PUB-2007-013

\footnotetext{
${ }^{4}$ For that purpose, a Monte Carlo simulation of $5 \mathrm{GeV}$ projective electrons is used.
} 